\title{
BMJ Open Development and validation of the OUTCoV score to predict the risk of hospitalisation among patients with SARS-CoV-2 infection in ambulatory settings: a prospective cohort study
}

To cite: Jacquerioz F, Baggio $\mathrm{S}$, Gayet-Ageron A, et al. Development and validation of the OUTCoV score to predict the risk of hospitalisation among patients with SARSCoV-2 infection in ambulatory settings: a prospective cohort study. BMJ Open 2021;11:e044242. doi:10.1136/ bmjopen-2020-044242

- Prepublication history and additional supplemental material for this paper are available online. To view these files, please visit the journal online (http://dx.doi.org/10.1136/ bmjopen-2020-044242).

Received 27 August 2020 Accepted 12 May 2021

\section{Check for updates}

(C) Author(s) (or their employer(s)) 2021. Re-use permitted under CC BY-NC. No commercial re-use. See rights and permissions. Published by BMJ.

For numbered affiliations see end of article.

\section{Correspondence to} Dr Frederique Jacquerioz; Frederique.Jacquerioz@hcuge. ch

\section{ABSTRACT}

Objectives To develop and validate a rule-out prediction model for the risk of hospitalisation among patients with SARS-CoV-2 infection in the ambulatory setting to derive a simple score to determine outpatient follow-up.

Design Prospective cohort study.

Setting Swiss university hospital.

Participants 1459 individuals with a positive result for SARS-CoV-2 infection between 2 March and 23 April 2020.

Methods We applied the rule of 10 events per variable to construct our multivariable model and included a maximum of eight covariates. We assessed the model performance in terms of discrimination and calibration and performed internal validation to estimate the statistical optimism of the final model. The final prediction model included age, fever, dyspnoea, hypertension and chronic respiratory disease. To develop the OUTCoV score, we assigned points for each predictor that were proportional to the coefficients of the regression equation. Sensitivity, specificity, positive and negative likelihood ratios were estimated, including positive and negative predictive values in different thresholds.

Main outcome measure The primary outcome was COVID-19-related hospitalisation.

Results The OUTCoV score ranged from 0 to 7.5 points. The two threshold parameters with optimal rule-out and rule-in characteristics for the risk of hospitalisation were 3 and 5.5 , respectively. Outpatients with a score $<3$ (997/1459; 68.3\%) had no follow-up as at low risk of hospitalisation (1.8\%; $95 \% \mathrm{Cl} 1.1$ to 2.8 ). For a score $\geq 5.5$ (20/1459; $1.4 \%)$, the hospitalisation risk was higher (30\%; $95 \% \mathrm{Cl} 11.9$ to 54.3 ).

Conclusions The OUTCoV score allows to rule out twothirds of outpatients with SARS-CoV-2 infection presenting a low hospitalisation risk and to identify those at high risk that require careful follow-up to assess the need for hospitalisation. The model provides a simple decisionmaking tool for an effective allocation of resources to maintain quality care for outpatient populations.

\section{Strengths and limitations of this study}

- To our knowledge, prediction model for the risk of hospitalisation are scarce among patients presenting with symptoms of COVID-19 in ambulatory settings.

- Our prediction model has a very good discrimination capacity, especially for identifying patients that do not need a close follow-up and the overall calibration of the OUTCoV score is almost perfect.

- This easy-to-use score provides clinicians with a simple decision-making tool in prehospital care management while ensuring quality of care for vulnerable populations, even in low-resource settings.

- The low number of outcome events leads to an overprediction of the hospitalisation risk for high-risk outpatients. This also limited the number of variables in the scoring model. Thus, the model needs retrospective and prospective external validation in diverse sets of outpatients and larger cohorts.

- Variables such as body mass index, socioeconomic status, oxygen saturation or chest $X$-ray were not systematically collected and need to be further investigated as risk factors for hospitalisation and added in the model.

\section{INTRODUCTION}

COVID-19 was declared a pandemic by the WHO on 11 March 2020. Based on the available observations, clinicians first considered the disease as restricted to the respiratory tract and the initial case definition focused on the presence of upper and/or lower respiratory tract symptoms associated with fever. ${ }^{1}$ As the outbreak rapidly extended worldwide, it became apparent that COVID-19 included a wider spectrum, ranging from asymptomatic or mild self-limited infections to severe pneumonia and clinical presentations beyond respiratory symptoms. ${ }^{2-12}$ 
As of May 2021, >160 million cases of COVID-19 have been reported worldwide, although the majority do not require hospitalisation. However, it remains essential to grasp the whole clinical picture, including symptoms and predictive factors of disease progression among the outpatient population, to identify those that can safely stay isolated at home with minimum follow-up. This distinction is particularly important when the number of patients increases exponentially, or countries experience new waves of cases. To date, COVID-19 clinical and epidemiological characteristics have been mainly reported among patients hospitalised with pneumonia ${ }^{713-15}$ and only a few studies have focused on risk factors associated with the clinical outcome and disease progression, including a comparison of the various degrees of severity observed during hospitalisation. ${ }^{511}$

This study aimed to develop and validate a prediction model to derive a simple ambulatory score (OUTCoV score) based on symptoms, age and comorbidities that could be used among outpatients with SARS-CoV-2 infection to help clinicians decide on an appropriate follow-up of patients isolated at home.

\section{METHODS}

\section{Study design, setting and participants}

We conducted a single-centre, prospective, cohort study of outpatients presenting to the SARS-CoV-2 testing centres at Geneva University Hospitals (Hôpitaux Universitaires de Genève (HUG), Geneva, Switzerland) between 2 March and 23 April 2020, corresponding to the epidemic first wave. HUG is a 2000-bed teaching hospital and one of the largest in Europe, serving a population of approximately 500000 residents. Four dedicated SARS-CoV-2 testing centres within the existing outpatient facilities were set up at the end of February 2020. Patients presenting with severe signs of infection were reoriented to the HUG emergency room. All COVID-19-related hospitalisations (1088 at 23 April 2020) in the canton of Geneva were centralised at HUG.

The testing strategy followed the recommendations of the Swiss national health authorities and targeted all persons with respiratory symptoms with or without fever. After testing positive for SARS-CoV-2 infection, all patients who did not immediately present hospitalisation criteria were isolated at home for at least 10 days and followed-up through regular telephone calls by the local health authorities. An in-person medical evaluation was proposed for those who presented symptoms of disease progression, either at home or at the emergency care department, depending on severity.

Patients were included if they attended the HUG testing centres during the study period and had a positive SARS-CoV-2 test. Exclusion criteria were as follows: (1)<16 years old; (2) history of a positive test; (3) refusal of consent to use their data for research purposes and (4) patients requiring immediate hospitalisation.

\section{Variables collected and predictors}

Demographic, epidemiological and clinical data were obtained through standardised self-report questionnaires. Each questionnaire was then reviewed and completed by a nurse, a trained medical student or a doctor. Symptoms included runny nose, sore throat, dry or productive cough, fever (anamnestic or measured), muscle pain, chills and a free text to collect other symptoms. The latter were then recoded under headache, loss of smell or taste, fatigue, difficulty breathing, abdominal complaints and thoracic discomfort and added in the more recent versions of the questionnaires. Date of symptom onset was also collected. Comorbidities were defined accordingly to international and Swiss recommendations and included the following: hypertension, chronic respiratory diseases (ie, severe asthma and chronic obstructive pulmonary disease), diabetes, cardiovascular disease (ie, coronary heart disease, ancient myocardial infarction, stroke, valvular heart disease, heart failure, atrial fibrillation and severe arrhythmia) and immunosuppression status (ie, solid organ transplant, cancer including oncohematologic, immunosuppressive treatment and immunodeficiency (HIV)).

\section{Primary outcome}

COVID-19-related hospitalisation was defined as the primary outcome. Hospitalisation criteria included a CURB-65 (pneumonia risk) score $\geq 2$; new dependence on or an increased need of oxygen; sustained tachypnoea; poorly controlled comorbidities; deteriorated general condition or worsening disease. Hospitalisation was captured from patients' medical records up to 4 weeks after positive testing for all patients.

\section{Infection status}

SARS-CoV-2 infection was detected by reverse transcriptase (RT)-PCR performed on nasopharyngeal or oropharyngeal swabs obtained by either nurses or doctors following a standardised procedure and processed at the HUG virology laboratory, the Swiss national reference laboratory for SARS-CoV-2. RT-PCR was performed according to manufacturers' instructions on various platforms, including initially an in-house method using eMAG (bioMérieux, Marcy l'Etoile, France) and the Charité/ Berlin RT-PCR protocol, ${ }^{16}$ followed by the BD SARS-CoV-2 reagent kit for BD Max system (Becton Dickinson \& Co, Franklin Lakes, NJ, USA) and Cobas 6800 SARS-CoV-2 RT-PCR (Roche Diagnostics, Rotkreuz, Switzerland).

\section{Statistical analysis}

We used a fixed sample of all hospitalised patients $(n=80)$ among those who tested positive to SARS-CoV-2 infection by RT-PCR testing, but who did not require immediate hospitalisation. We applied the rule of at least 10 events per variable to construct our multivariable model and included a maximum of eight covariates. ${ }^{17}$ We first computed descriptive statistics for all variables (percentages and $\mathrm{n}$ for categorical variables, mean values and 
SDs for continuous variables), followed by the $95 \%$ CIs to estimate the prevalence of the main outcome (hospitalisation).

\section{Prediction model development}

All 1459 patients who tested positive by RT-PCR testing in the development cohort were included for variable selection and risk score. We tested whether symptoms (fever, chills, difficulty breathing, dry or productive cough, abdominal complaints, loss of smell or taste and thoracic discomfort), age, gender and comorbidities (diabetes, hypertension, chronic respiratory disease, cardiovascular disease and immunosuppressive status) predicted hospitalisation using simple logistic regression models. All variables were assessed in univariate analysis. We then predefined a list of variables to be included in a multivariable model based on a clinical perspective, but also to respect the conservative convention of at least 10 events per variable. ${ }^{17}$ We assessed departures from linearity by plotting the risk of hospitalisation against continuous variables (age). We selected the age categories on a clinical perspective $(<35,35-44,45-54,55-64$ and $\geq 65$ years $)$ and verified that the linear spline using these cut-offs best followed the lowest. We chose to include all the following variables: fever, chills, difficulty breathing, dry or productive cough, abdominal complaints, loss of smell or taste, thoracic discomfort, age in categories, gender, diabetes, hypertension, chronic respiratory disease, cardiovascular disease and/or immunosuppressive status. Then, we performed a stepwise backward procedure and removed all variables with $p \geq 0.05$ or confounding variables at the end, we verified the fit of the model by Hosmer-Lemeshow test.

\section{Model performance and validation}

We assessed the model performance in terms of discrimination and calibration. Discrimination was assessed with the C-statistic and the area under the receiver operating characteristic curve. ${ }^{18}$ Calibration was assessed as the difference between the mean observed and predicted probabilities and by plotting the observed outcome and predicted probabilities by decile of the predicted risk of hospitalisation including non-parametric smoothing. ${ }^{19}$ A calibration slope of 1 and an intercept of 0 indicated perfect calibration. Overall calibration was summarised by the ratio of the expected number of events and observed number of events $(\mathrm{E} / \mathrm{O})$ with an optimal value of $1, \mathrm{E}$ / $\mathrm{O}<1$ indicated underprediction and $>1$ overprediction.

Internal validation was performed to estimate the statistical optimism of the final model. We drew 200 bootstrapped samples of 1459 patients and developed a model in each sample, including variable selection. We then estimated the C-statistic in each sample and assessed the performance of each model in the original sample. Optimism was estimated as the mean of the difference between the C-statistic of the bootstrap sample and that of the original sample. We subtracted optimism from the C-statistic of the model developed in the original sample to obtain the optimism-corrected C-statistic. Finally, we performed an internal-external validation. ${ }^{19}$ We performed a crossvalidation procedure using four time periods (2-15 March 2020, 16-29 March 2020, 30 March-12 April 2020 and 13-23 April 2020). We left out one period in turn and developed models using the same predictors in the remaining periods and estimated discrimination and calibration in the omitted periods. Again, C-statistics, calibration slope and overall calibration for each period were pooled with random effects. We assessed heterogeneity with $\mathrm{I}^{2}$ statistics.

\section{Derivation of the OUTCoV score}

The final predictive model included age categories, clinical symptoms (fever and dyspnoea) and comorbidities (high blood pressure and chronic respiratory disease). To develop the OUTCoV score, we assigned points for each predictor that were proportional to the coefficients of the regression equation and rounded at 0.5 decimal. We assessed the accuracy, discrimination and calibration of the OUTCoV score. Accuracy was assessed using the Brier score. As the Brier score depends on the prevalence of the outcome, we also calculated the scaled Brier score to account for the baseline risk of hospitalisation. The scaled version ranges from $0 \%$ to $100 \%$ and indicates the degree of error in prediction. ${ }^{19}$ We estimated 'calibration-in-thelarge' as the difference between the mean predicted and observed probabilities and the ratio of the predicted and observed number of events. We also plotted the observed and predicted probabilities of death by decile of the score and with local regression based on the LOESS algorithm. ${ }^{19}$ We estimated the calibration intercept and slope of the calibration plot as a measure of the spread between the predicted and observed outcome. The sensitivity, specificity, positive and negative likelihood ratios of the OUTCoV score were estimated, including the positive and negative predictive values

To provide optimal values for the rule-in and rule-out. ${ }^{20}{ }^{21}$ All analyses were performed using STATA software (V.16.0; Stata Corp, College Station, TX, USA).

\section{Patient and public involvement}

Given the urgency and rapid progression of the COVID-19 pandemic, there was a need to disseminate information quickly and thus patients were not directly involved in the development, implementation or interpretation of this research study. Furthermore, this study was based on chart reviews, which were not accessible to non-medical or non-research personnel, limiting patient and public involvement.

\section{RESULTS}

\section{Participants}

Among the 7699 visits to the testing centres, 1487 (19.3\%) individuals tested positive for SARS-CoV-2 infection. A total of 28 visits were excluded as they corresponded to SARS-CoV-2-infected cases re-consulting after a first visit ${ }^{19}$ 
or they did not consent to the use of their data for research purposes. ${ }^{9}$ A total of 1459 participants were included in the analysis (mean age $41.8 \pm 13.3$ years; $57.8 \%$ (843) were women). On 23 April 2020, they represented 30.8\% (1459/4726) of the SARS-CoV-2-positive cases in the canton of Geneva. The remaining positive cases (69.2\%) corresponded to patients tested in private laboratories, clinics and nursing homes, or at the time of hospitalisation for severe conditions related or unrelated to COVID19. A total of $52.8 \%(771 / 1459)$ consulted during the first 3 days of symptom onset, $27.1 \%$ (395/1459) between 4 and 7 days and $14.4 \%(210 / 1459)$ after 7 days. A total of $1.4 \%(21 / 1459)$ did not have a date of symptom onset as they were asymptomatic, and the remaining $4.3 \%$ $(63 / 1459)$ were unable to give a date of symptom onset.

\section{Outcome data}

Eighty of 1459 participants were hospitalised for COVID-19 (5.5\%; $95 \%$ CI 4.4 to 6.8$)$ during the follow-up period. Mean age was $51.5 \pm 13.2$ years; $48.8 \%$ were women.

\section{Predictors of hospitalisation}

Symptoms and vulnerability factors significantly associated with hospitalisation are reported in table 1. An increased risk of hospitalisation was clearly associated with the number of risk factors present: one or more risk factors (OR 3.77 (95\% CI 2.38 to 5.94); $\mathrm{p}=0.001$ ); two or more risk factors (OR 5.87 (95\% CI 3.28 to 10.49); $\mathrm{p}<0.001)$ and three or more risk factors (OR $6.20(95 \%$ CI 2.55 to 15.06$) ; \mathrm{p}<0.001$ ).

\section{Prediction model of hospital admission}

The final prediction model included five covariates (table 2). The performance of the model developed showed good discrimination with a C-statistic of 0.79 (0.75 to 0.84 ). Calibration was good with no differences between observed and predicted hospitalisation, except for high-risk patients $(20 / 1459)$ in whom the risk was overestimated above a predicted probability of 0.3 (figure 1 ). Bootstrap resampling showed a small model optimism of 0.0256 and gave an optimism-corrected C-statistic of 0.7697 that was slightly changed. At internal-external validation, the C-statistic ranged from 0.75 to 0.89 . The pooled C-statistic was 0.81 ( 0.75 to 0.88$)$ with no heterogeneity across the four periods $\left(\mathrm{I}^{2}=51.7 \%, \mathrm{p}=0.102\right)$. The pooled calibration slope was 0.65 (0.35 to 0.96$)$. The calibration slope and overall calibration showed heterogeneity, especially for periods 1 and 4, due to unequal number of events across the four periods $\left(\mathrm{I}^{2}=69.3 \%\right.$ and $73.2 \%$ ( $\mathrm{p}=0.021$ and $\mathrm{p}=0.011)$, respectively).

\section{The OUTCoV score}

We created the OUTCoV score as follows: fever, 1.5 points; dyspnoea, 1 point; chronic respiratory disease, 1 point and hypertension, 1.5 points. For age categories (reference $<35$ years): $35-44$ years, 1 point; $45-54$ years, 1.5 points; $55-64$ years, 2 points and $\geq 65$ years, 2.5 points. The total score ranged from 0 to 7.5 points. The performance of the score is shown in online supplemental table
S1. The scaled Brier score was $18 \%$. The calibration curve showed overprediction in high-risk patients, similar to the development prediction model (figure 2). The calibration intercept was close to $0(0.0064)$ with a calibration slope of 0.88 ; overall calibration was perfect (online supplemental table S1). The sensitivity and specificity, positive and negative likelihood ratios, and positive and negative predictive values at different thresholds of the OUTCoV score are shown in table 3. We chose two cutoff (3 and 5.5) which optimised the decision rules. We added a specific table presenting the negative predictive value for score $<3$ and positive predictive value for score between 3 and 5.5, and score $\geq 5.5$ (table 4 ).

\section{DISCUSSION}

In this study, we constructed and internally validated a simple clinical scoring model-the OUTCoV scoreto predict hospitalisation of individuals with COVID-19 using data from an ambulatory cohort of newly diagnosed SARS-CoV-2-infected patients in the canton of Geneva (Switzerland), which was severely hit by the COVID-19 first wave. ${ }^{22}$ The COVID-19 pandemic has resulted in a rapidly increasing number of infected patients isolated at home. It is thus crucial to adapt prehospital case management and develop strategies to safely keep patients at home and promptly identify those that may need hospitalisation.

Our scoring model ranges from 0 to 7.5 points based on variables independently predictive of hospitalisation. To enhance its practical implementation, we categorised the model into the following three groups according to the level of risk of being hospitalised: low (0-2.5 points); intermediate (3-5 points) and high (5.5-7.5 points). We chose the model that best identified patients that have the least chance of developing complications leading to hospitalisation (rule-out risk). This is particularly important as an essential part of the strategy to avoid overwhelming health systems is to encourage home isolation of SARS-CoV-2infected patients with mild symptoms, without sacrificing quality of care. In the selected model, a low risk translated as a $1.8 \%$ chance of being hospitalised and corresponded to $68 \%$ of our study population. By contrast, a high risk meant a $30 \%$ chance of being hospitalised, with $1.4 \%$ of our patients in this category. Although the model overestimated the risk of hospitalisation in the latter group, this represented a small number of patients who would have been more regularly contacted to assess their health condition.

The OUTCoV score model highlights the importance of focusing on individuals with fever, dyspnoea, older age and comorbidities (particularly hypertension and chronic respiratory disease). Fever and dyspnoea as predicting symptoms of hospitalisation is consistent with the meta-analysis of Zheng et $a l,^{23}$ which reported the same symptoms for disease progression during hospitalisation among 13 studies looking at risk factors for critical and fatal COVID-19. Guan $e ~_{a l}{ }^{4}$ reported fever in almost all patients with pneumonia during hospitalisation, but 
Table 1 Associations between symptoms, age, comorbidities and hospitalisation

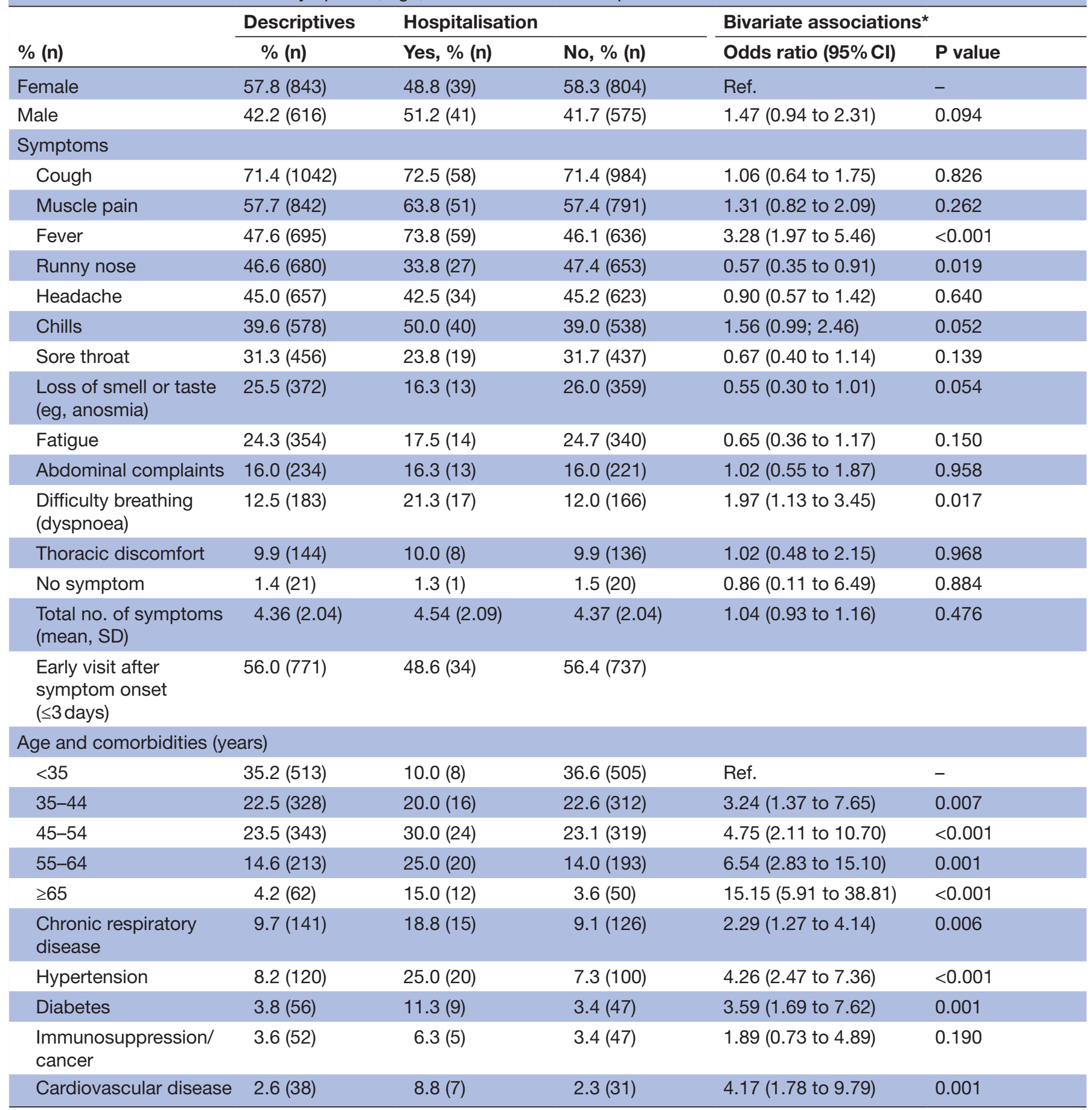

*Simple logistic regressions: outcome: hospitalisation.

only half had fever at symptom onset. Therefore, when present, fever should not be minimised, even in patients not so severely ill as to suggest a risk of complications. Age $\geq 65$ years was the variable most strongly associated with hospitalisation.

Regarding comorbidity and adjustment for the confounding effect of age, only hypertension remained significant and chronic respiratory diseases was only marginally significant. We chose to keep both in the model and did not include other comorbidities. However, recent studies on inpatients with COVID-19 have reported that all comorbidities were significantly more prevalent in critically ill patients and associated with adverse outcomes. ${ }^{23}$ The discrepancy with our findings may be due to the limited number of hospitalisations. Overall, we can assume that comparable comorbidities predict disease progression among outpatients, thus leading to hospitalisation, and among inpatients (leading to adverse outcomes). 
Table 2 Prediction model for the risk of hospitalisation among patients newly diagnosed with SARS-CoV-2 infection in ambulatory settings

\begin{tabular}{|c|c|c|}
\hline & \multicolumn{2}{|c|}{ Multivariable associations* } \\
\hline & Odds ratio $(95 \% \mathrm{Cl})$ & $P$ value \\
\hline $\begin{array}{l}\text { Age (reference <35 } \\
\text { years) }\end{array}$ & & $<0.001$ \\
\hline $35-44$ & 3.16 (1.32 to 7.58$)$ & 0.010 \\
\hline $45-54$ & 4.32 (1.88 to 9.93$)$ & 0.001 \\
\hline $55-64$ & $5.51(2.31$ to 13.14$)$ & $<0.001$ \\
\hline$\geq 65$ & 9.26 (3.34 to 25.68$)$ & $<0.001$ \\
\hline $\begin{array}{l}\text { Dyspnoea } \\
\text { (reference none) }\end{array}$ & 2.52 (1.39 to 4.54$)$ & 0.002 \\
\hline $\begin{array}{l}\text { Fever (reference } \\
\text { none) }\end{array}$ & 3.44 (2.02 to 5.84$)$ & $<0.001$ \\
\hline $\begin{array}{l}\text { Hypertension } \\
\text { (reference none) }\end{array}$ & 2.90 (1.57 to 5.36$)$ & 0.001 \\
\hline $\begin{array}{l}\text { Chronic respiratory } \\
\text { diseases (reference } \\
\text { none) }\end{array}$ & $2.23(1.19$ to 4.16$)$ & 0.012 \\
\hline
\end{tabular}

*Logistic regression. AUC of the model: 0.7954 (0.74590.8449). Hosmer-Lemeshow: $\mathrm{p}=0.097$.

AUC, area under the curve.

Therefore, this aspect has to be weighted when using the score and further studied in larger populations.

\section{Implications for clinical practice}

Based on the risk of hospitalisation, the model calls for an adapted strategy of patient follow-up to assess their health condition during the first 10 days of disease. We propose that individuals in the low-risk group be given the option of calling a health professional or visit an emergency care

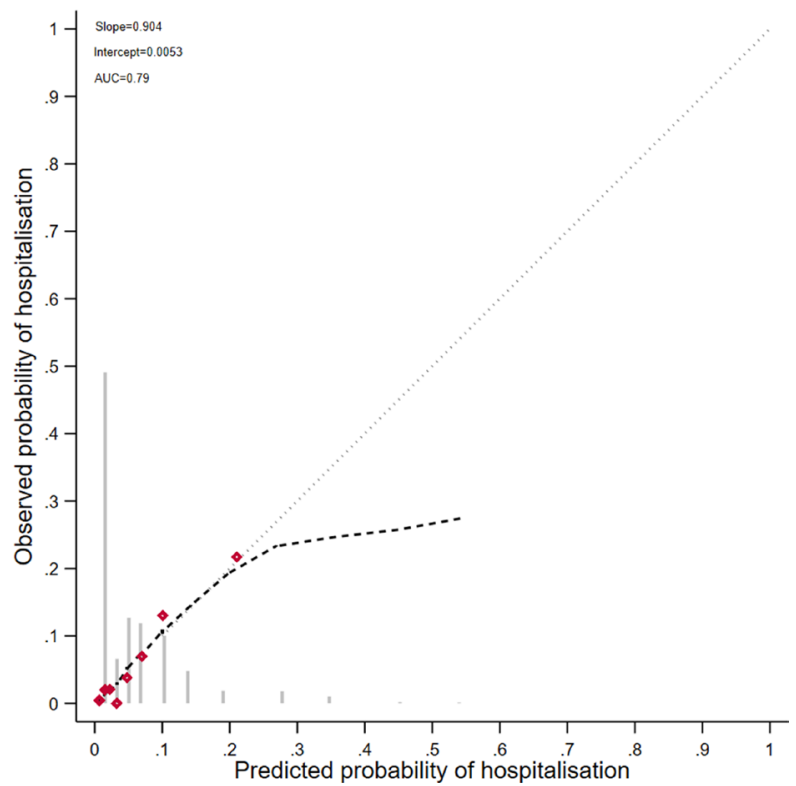

Figure 1 Calibration curve of the development prediction model. AUC, area under the curve.

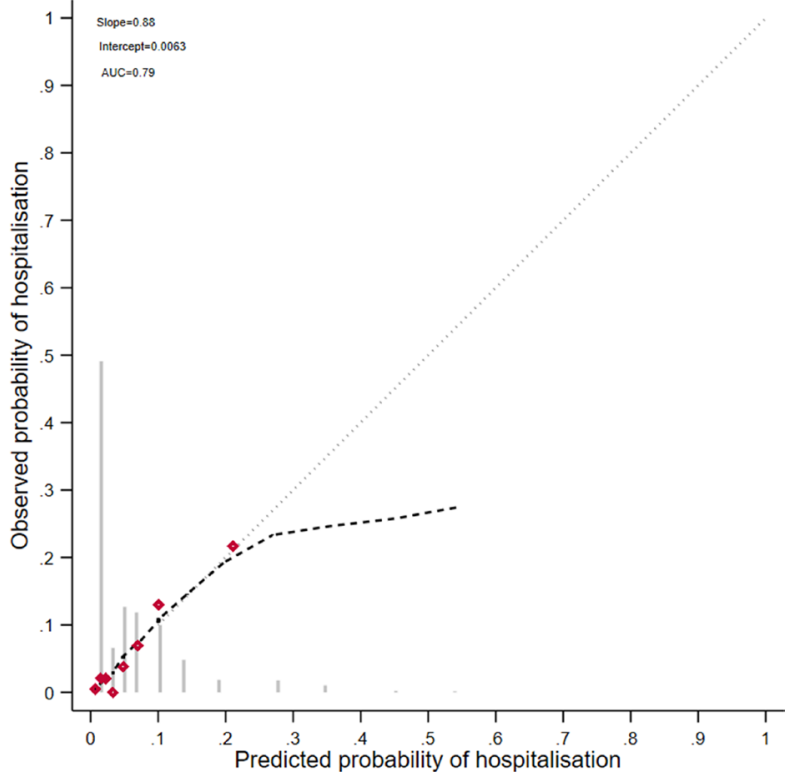

Figure 2 Calibration of the the OUTCoV score. AUC, area under the curve.

facility if their condition worsens. Individuals in the highrisk group require a very close ambulatory monitoring and will benefit the most from daily or every other day calls by health professionals to evaluate how their condition evolves. with in-person visits when necessary. In that group, follow-up could last longer than 10 days. Individuals with an intermediate risk could be called every 3-4 days. They could also benefit from a follow-up system, such as the one developed by the HUG primary care service entitled the 'Patient-Reported Outcome COVID19' (PRO-COVID),${ }^{24}$ with the aim to facilitate the dissemination and sharing of COVID processes for ambulatory care centres worldwide. In brief, PRO-COVID allows the patient to report his/her symptoms on a daily basis using an electronic secured platform. If serious symptoms are reported, the patient is called for a medical evaluation. The score can be adjusted to the patient's disease progression, meaning that the same patient might change group at each evaluation.

All variables in the OUTCoV scoring model are easy to collect during the initial medical encounter and do not require laboratory work. The score could also be used as a self-assessment tool on a digital app, with the advantage of providing patients and clinicians with an easy-to-use tool for at-home patient monitoring. This is extremely useful when the number of patients exceeds the health system resources and follow-up services need to be focused on vulnerable patients at most risk of complications. In addition, OUTCoV can be used in addition to providing continuity of care and adaptation of treatments for chronic diseases that are crucial elements to help prevent detrimental outcomes of SARS-CoV-2 infection in the absence of a curative treatment for COVID-19. Importantly, the scoring system can be used in all settings and populations and can be a useful decision-making tool in countries with limited resources and an exponential number of cases. 


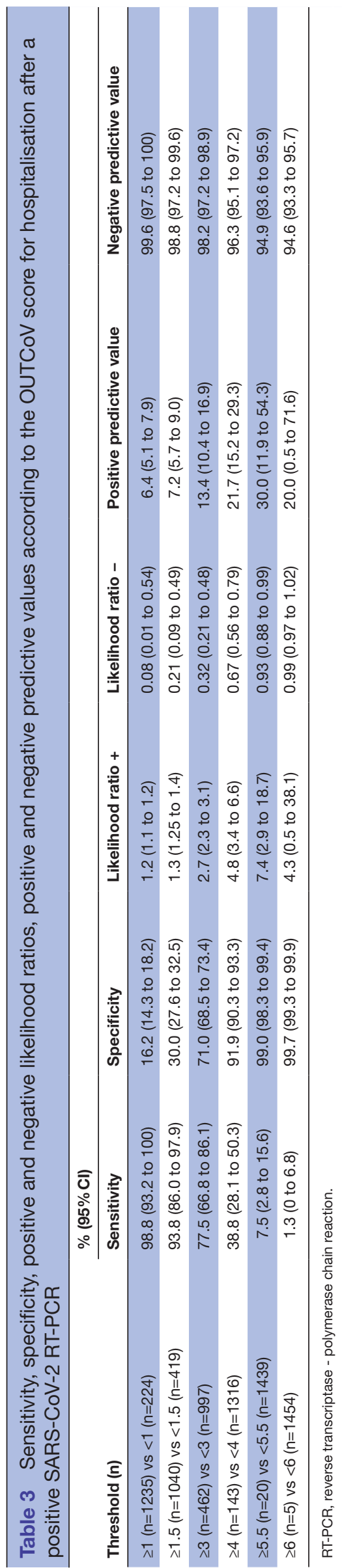

Table 4 Positive and negative predictive values according to OUTCoV score for hospitalisation after positive SARSCoV-2 RT-PCR at the two chosen cut-offs

\begin{tabular}{lll}
\hline Threshold $(\mathrm{n})$ & $\begin{array}{l}\text { Positive predictive } \\
\text { value }\end{array}$ & $\begin{array}{l}\text { Negative predictive } \\
\text { value }\end{array}$ \\
\hline$<3(\mathrm{n}=997)$ & - & $98.2(97.2$ to 98.9$)$ \\
$\geq 3$ and $<5.5(\mathrm{n}=442)$ & $12.7(9.7$ to 16.1$)$ & - \\
$\geq 5.5(n=20)$ & $30.0(11.9$ to 54.3$)$ & - \\
\hline
\end{tabular}

The area under the ROC curve for this score was 0.7932 (95\% $\mathrm{Cl} 0.7436$ to 0.8429 ).

ROC, receiver operating characteristic; RT-PCR, reverse transcriptase - polymerase chain reaction.

\section{Strengths and limitations of this study}

Our study has unique strengths. To our knowledge, prediction models for the risk of hospitalisation are scarce. During the first wave, most countries applied a lockdown period to preserve their care facilities from an uncontrolled patient flow and saturation of the healthcare system. There is a pressing need to have simple and useful tools to personalise outpatient care management and optimise follow-up strategies. Our prediction model has a very good discrimination capacity and overall calibration of the OUTCoV score is almost perfect. Although external validation of the model was not performed, we conducted an appropriate internal validation with bootstrapping, completed by an internalexternal validation. ${ }^{19}$ All these validations ensured that our score had a validation per se.

The study has some limitations. First, the number of outcome events was low, leading to an overprediction of the hospitalisation risk for high-risk outpatients. This also limited the number of variables in the scoring model. Thus, the model needs retrospective and prospective external validation in diverse sets of outpatients and larger cohorts. However, the score shows a very good discrimination for identifying patients that do not need a close follow-up and is particularly useful for settings with an exponential number of infected individuals and widespread testing strategies that capture milder cases. Second, variables such as body mass index, socioeconomic status, oxygen saturation or chest X-ray were not systematically collected and need to be further investigated as risk factors for hospitalisation and added in the model. Moreover, we used data that were pragmatically retrieved in a very simple questionnaire submitted to patients when they waited for SARS-CoV-2 screening. This questionnaire was not preliminary validated using mixed-methods design such as for the Remote COVID-19 Assessment in Primary Care (RECAP-V0) ${ }^{25}$

Third, participants non-resident in Geneva could have been hospitalised elsewhere. However, we ran sensitivity analyses excluding individuals who lived outside the canton of Geneva and the findings were similar (online supplemental tables S2 and S3) Finally, approximately half of the reported SARS-CoV-2-infected individuals in Geneva canton were diagnosed in private laboratories or clinics across the city. The choice of where to be tested was certainly based 
on convenience and proximity and we do not expect any major difference between those tested at the HUG testing centres versus elsewhere.

\section{CONCLUSION}

The OUTCoV score helps to rule-out two-thirds of outpatients with SARS-CoV-2 infection and a low risk of hospitalisation. This allows to focus on a small number of high-risk outpatients who may require careful follow-up to timely identify the need for hospitalisation. As part of a pre-hospital case management strategy, the OUTCoV scoring model provides a simple decision-making tool that leads to an effective allocation of resources and ensures quality care for populations at risk of complications.

\section{Author affiliations}

${ }^{1}$ Division of Tropical and Humanitarian Medicine, Geneva University Hospitals, Geneva, Switzerland

${ }^{2}$ Center for Emerging Viral Diseases, Geneva University Hospitals, Geneva, Switzerland

${ }^{3}$ Division of Primary Care, Geneva University Hospitals, Geneva, Switzerland ${ }^{4}$ Division of Prison Health, Geneva University Hospitals, Geneva, Switzerland ${ }^{5}$ Division of Clinical Epidemiology, Department of Health and Community Medicine, Geneva University Hospitals \& University of Geneva, Geneva, Switzerland ${ }^{6}$ Division of Infectious Diseases, Geneva University Hospitals, Geneva, Switzerland ${ }^{7}$ Virology Laboratory, Geneva University Hospitals, Geneva, Switzerland

${ }^{8}$ Medical and Quality Directorate, Geneva University Hospitals, Geneva, Switzerland

Twitter Frederique Jacquerioz @FrederiqueJ and Nathalie Vernaz @vernaz_info

Acknowledgements We thank the medical students who performed data entry, in particular Lucas Broc, the nursing staff at the testing centres, and the medical team of the Geneva health authorities. We also thank all the participants of the study.

Contributors FJ, SB, HS and LK: concept and design. FJ, SB, AG-A and SY: acquisition, analysis and data interpretation. FJ: drafting of the manuscript. All authors (FJ, SB, AG-A, FC, LG, IG, LK, NV, SY, JS and HS): critical revision of the manuscript for important intellectual content. SB and AG-A: statistical analysis. LK and FC: supervision and guarantors of the study.

Funding The authors have not declared a specific grant for this research from any funding agency in the public, commercial or not-for-profit sectors.

Competing interests None declared.

Patient consent for publication Not required.

Ethics approval The study was approved by the HUG institutional ethics board (no. 2020-00813). Patients who refused the use of their data were removed from the dataset.

Provenance and peer review Not commissioned; externally peer reviewed.

Data availability statement Data are available upon reasonable request. The authors commit to making the relevant anonymised patient-level data available on reasonable request.

Supplemental material This content has been supplied by the author(s). It has not been vetted by BMJ Publishing Group Limited (BMJ) and may not have been peer-reviewed. Any opinions or recommendations discussed are solely those of the author(s) and are not endorsed by BMJ. BMJ disclaims all liability and responsibility arising from any reliance placed on the content. Where the content includes any translated material, BMJ does not warrant the accuracy and reliability of the translations (including but not limited to local regulations, clinical guidelines, terminology, drug names and drug dosages), and is not responsible for any error and/or omissions arising from translation and adaptation or otherwise.

Open access This is an open access article distributed in accordance with the Creative Commons Attribution Non Commercial (CC BY-NC 4.0) license, which permits others to distribute, remix, adapt, build upon this work non-commercially, and license their derivative works on different terms, provided the original work is properly cited, appropriate credit is given, any changes made indicated, and the use is non-commercial. See: http://creativecommons.org/licenses/by-nc/4.0/.

\section{ORCID iDs}

Frederique Jacquerioz http://orcid.org/0000-0002-6957-0920

Stéphanie Baggio http://orcid.org/0000-0002-5347-5937

Angele Gayet-Ageron http://orcid.org/0000-0002-6164-9693

\section{REFERENCES}

1 WHO. Report of the WHO-China joint mission on coronavirus disease 2019 (COVID-19), 2020.

2 Lescure F-X, Bouadma L, Nguyen D, et al. Clinical and virological data of the first cases of COVID-19 in Europe: a case series. Lancet Infect Dis 2020;20:697-706.

$3 \mathrm{Ng}$ O-T, Marimuthu K, Chia P-Y, et al. SARS-CoV-2 infection among travelers returning from Wuhan, China. N Engl J Med 2020;382:1476-8.

4 Vetter P, Vu DL, L'Huillier AG, et al. Clinical features of covid-19. BMJ 2020;369:m1470.

5 Yang X, Yu Y, Xu J, et al. Clinical course and outcomes of critically ill patients with SARS-CoV-2 pneumonia in Wuhan, China: a singlecentered, retrospective, observational study. Lancet Respir Med 2020;8:475-81.

6 Young BE, Ong SWX, Kalimuddin S, et al. Epidemiologic features and clinical course of patients infected with SARS-CoV-2 in Singapore. JAMA 2020;323:1488.

7 Wang D, Hu B, Hu C, et al. Clinical characteristics of 138 hospitalized patients with 2019 novel coronavirus-infected pneumonia in Wuhan, China. JAMA 2020;323:1061.

8 Bonow RO, Fonarow GC, O'Gara PT, et al. Association of coronavirus disease 2019 (COVID-19) with myocardial injury and mortality. JAMA Cardiol 2020;5:751.

9 Inciardi RM, Lupi L, Zaccone G, et al. Cardiac involvement in a patient with coronavirus disease 2019 (COVID-19). JAMA Cardiol 2020;5:819.

10 Mao L, Jin H, Wang M, et al. Neurologic manifestations of hospitalized patients with coronavirus disease 2019 in Wuhan, China. JAMA Neurol 2020;77:683.

11 Zhou F, Yu T, Du R, et al. Clinical course and risk factors for mortality of adult inpatients with COVID-19 in Wuhan, China: a retrospective cohort study. Lancet 2020;395:1054-62.

12 Shi S, Qin M, Shen B, et al. Association of cardiac injury with mortality in hospitalized patients with COVID-19 in Wuhan, China. JAMA Cardiol 2020;5:802.

13 Chen N, Zhou M, Dong X, et al. Epidemiological and clinical characteristics of 99 cases of 2019 novel coronavirus pneumonia in Wuhan, China: a descriptive study. Lancet 2020;395:507-13.

14 Guan W-J, Ni Z-Y, Hu Y, et al. Clinical characteristics of coronavirus disease 2019 in China. N Engl J Med 2020;382:1708-20.

15 Huang C, Wang Y, Li X, et al. Clinical features of patients infected with 2019 novel coronavirus in Wuhan, China. Lancet 2020;395:497-506.

16 Corman VM, Landt O, Kaiser M, et al. Detection of 2019 novel coronavirus (2019-nCoV) by real-time RT-PCR. Euro Surveill 2020;25.

17 Vittinghoff E, McCulloch CE. Relaxing the rule of ten events per variable in logistic and Cox regression. Am J Epidemiol 2007;165:710-8.

18 Royston P, Altman DG. Visualizing and assessing discrimination in the logistic regression model. Stat Med 2010;29:2508-20.

19 Steyerberg EW, Vickers AJ, Cook NR, et al. Assessing the performance of prediction models: a framework for traditional and novel measures. Epidemiology 2010;21:128-38.

20 Pauker SG, Kassirer JP. The threshold approach to clinical decision making. N Engl J Med 1980;302:1109-17.

21 Peng P, Coyle A. Likelihood ratios for the emergency physician. Acad Emerg Med 2018. [Epub ahead of print: 26 Apr 2018]. doi:10.1111/ acem. 13439

22 FOPH. New coronavirus: situation in Switzerland, 2020.

23 Zheng Z, Peng F, Xu B, et al. Risk factors of critical \& mortal COVID-19 cases: A systematic literature review and meta-analysis. $J$ Infect 2020;81:e16-25. doi:10.1016/j.jinf.2020.04.021

24 Covicare24, 2020. Available: https://www.covicare24.com/ [Accessed may 28, 2021].

25 Greenhalgh T, Thompson P, Weiringa S, et al. What items should be included in an early warning score for remote assessment of suspected COVID-19? qualitative and Delphi study. BMJ Open 2020;10:e042626. 